\title{
The Association Between Proton Pump Inhibitors and Incident Asthma in Patients with Coronary Artery Disease: A Population-Based Cohort Study
}

\section{Tsung-Kun Lin}

Taoyuan Armed Forces General Hospital

Jing-Yang Huang

Chung Shan Medical University

Chin-Feng Tsai

Chung Shan Medical University Hospital

Lung-Fa Pan

Central Taiwan University of Science and Technology

Gwo-Ping Jong ( $\nabla$ cgp8009@yahoo.com.tw)

Chung Shan Medical University Hospital

\section{Research Article}

Keywords: Proton pump inhibitors, coronary artery disease, asthma, gastroesophageal reflux disease

Posted Date: July 8th, 2021

DOI: https://doi.org/10.21203/rs.3.rs-671612/v1

License: (c) (1) This work is licensed under a Creative Commons Attribution 4.0 International License. Read Full License 


\section{Abstract}

Observational studies have found a significant association between acid-suppressive drug use and incident asthma. However, the association between proton pump inhibitors (PPIs) and incident asthma in patients with coronary artery disease (CAD) is unclear. Thus, this study assessed the association between PPI use and incident asthma in patients with CAD. We conducted a retrospective cohort study using the National Health Insurance Research Database in Taiwan from 2004 to 2013. Each patient who took PPIs was assigned to the PPI group, whereas 1:1 sex-, age-, and drug index date-matched randomly selected patients without PPI prescription were assigned to the non-PPI group. We analyzed the risk of incident asthma using Cox proportional hazard regression models, including sex, age, urbanization, low income, and comorbidities. Sensitivity and subgroup analyses were also conducted. A total of 8499 cases were identified as PPI-treated patients, and 8,499 subjects were included in the control group of PPI non-users. After adjusting for sex, age, urbanization, low income, and comorbidities, PPI user was associated with a 1.18-fold (HR: 1.18; 95\% Cl: 1.05-1.34) increase for incident asthma in patients with CAD. We concluded that PPI use increased the risk of incident asthma in patients with CAD. The risk of incident asthma should be considered when weighing the benefits and risks of PPI and aspirin treatment in patients with CAD in clinical practice.

\section{Introduction}

Asthma is a common disease among children. ${ }^{1}$ In 2016 , WHO estimated 417,918 deaths due to asthma at the global level and 24.8 million disability-adjusted-life-years attributable to asthma ${ }^{2}$. Numerous comorbidities can be associated with asthma and influence its clinical expression, but their specific influence remains to be characterized. Frequently contributing comorbid conditions reported in asthmatic patients include rhinitis, sinusitis, gastroesophageal reflux disease (GERD), obstructive sleep apnea, hormonal disorders, and psychopathologies, although other conditions, sometimes without an evident link with asthma, are also highly prevalent in asthmatic patients ${ }^{3}$.

Several studies have investigated the efficacy of different PPIs on asthma outcomes through randomized controlled trials (RCTs) ${ }^{4-8}$. Some studies have indicated that symptoms, lung function, or both can be improved with the treatment of acid reflux ${ }^{7-9}$; others have not demonstrated measurable improvement with acid suppression ${ }^{4,10,11}$. A Cochrane Library systematic review published in 2003 examined the effects of several antireflux treatments on asthma outcomes in children and adults ${ }^{12}$. The most recent systematic review, published in 2009, studied whether or not treatment of GERD with PPIs improves asthma symptoms in children ${ }^{13}$. Both studies were limited by small-scale RCTs using PPIs with conflicting results and provided no definitive recommendations regarding the use of PPIs for patients with asthma. The meta-analysis showed that prenatal exposure to acid-suppressive drugs, such as histamine H2-receptor antagonists and PPIs, is associated with an increased risk of childhood asthma. The evidence suggests that prenatal, maternal, acid-suppressive drug use is associated with an increased risk of childhood asthma ${ }^{14}$. 
Coronary artery disease (CAD) is one of the major cardiovascular diseases affecting the global human population ${ }^{15}$. Daily low-dose aspirin is recommended for the prevention of cardiovascular events in patients with CAD, and PPI is recommended to prevent or treat aspirin-associated gastrointestinal injury ${ }^{16-18}$. However, the association between PPIs use and incident asthma in patients with CAD remains unclear till now. Therefore, we examined the risk for incident asthma associated with PPI use in patients with CAD in the National Health Insurance Research Database (NHIRD) of Taiwan.

\section{Results}

Demographics. We identified 25,082 CAD patients with new diagnosed peptic ulcer or GERD, of whom we excluded 2312 who had asthma before 2004 and 637 dead during the follow-up period. Thus, we assessed data for 8,499 PPI users and 13,504 non-PPI users. After 1:1 age, sex, and drug index date, 8,499 non-PPI users were used for analyzing the relationship between the use of PPI and incident asthma (Figure 1). Characteristics at baseline are shown in Table 1. Most subjects were 20-60 years of age (71.21\% of PPI users and $70.99 \%$ of non-PPI users). Patients with PPI use had higher rates of comorbid hypertension ( $27.10 \%$ versus $25.87 \%)$, diabetes mellitus ( $13.95 \%$ versus $10.77 \%)$, stroke $(8.38 \%$ versus $5.31 \%$ ), pneumonia (4.47\% versus $2.29 \%$ ), and cancer (6.18\% versus $3.36 \%$ ) than the controls (all $p<$ 0.05). The follow-up periods were 719,322 person-months in the PPI group and 716,289 person-months in the non-PPI group.

The association between PPI use and incident asthma. Table 2 shows 706 asthma events ( 387 for the PPI cohort and 319 for the non-PPI cohort). The incidence was 1.21 -fold higher $(95 \% \mathrm{Cl}, 1.06-1.36)$ in the PPI cohort than in the non-PPI cohort (5.38 vs. 4.45 per 10,000 person-months), with an adjusted HR of 1.18 (95\% Cl, 1.05-1.34) after controlling for sex, age, urbanization, low income, and comorbidities. Kaplan-Meier method analysis showed that the cumulative probability of developing asthma from 2004 to 2013 follow-up period showed significant differences between the PPI and non-PPI cohorts (log-rank test, $p<0.0001$; Figure 2).

Sensitivity and subgroup analysis. Sensitivity analysis for the multiple Cox regression model confirmed the risk of incident asthma in the patients with PPI use when assessed using IPTW and propensity score matching (Table 3). In the subgroup analysis, the risk of asthma was significantly greater in the patients with male and female with PPI use than in patients without PPI use (aHR: 1.20, 95\% Cl: 1.06-1.41 versus aHR: 1.15, 95\% Cl: 1.01-1.36, respectively) (Table 4). However, the differences between these comorbidities were not differ among patients with hypertension, diabetes mellitus, hyperlipidemia, stroke, urticaria, atopic dermatitis, allergic rhinitis, pneumonia, cancer, or depression (Table 4).

\section{Discussion}

This large population-based cohort study found PPI use increased a 1.18-fold risk of asthma development in patients with CAD. However, subgroup analysis revealed no association between PPI use and the risk of incident asthma compared with non-PPI use in patients with hypertension, diabetes 
mellitus, hyperlipidemia, stroke, urticaria, atopic dermatitis, allergic rhinitis, pneumonia, cancer, or depression.

A significantly higher prevalence of asthma has been previously reported in patients with PPI use as compared with patients without PPI use, particularly in children ${ }^{19-22}$. Similarly, a recent study in children after high-dimensional propensity score matching has revealed a higher risk of incident asthma with PPI use comparable with non-PPI use (HR, 1.48; $95 \% \mathrm{Cl}, 1.41-1.55)^{22}$. Wang et al. conducted a population-based registry study in Sweden, which included 17,740 children with follow-up between January 2007 and June 2016. ${ }^{22}$ They used data from nationwide Swedish registries, with health care and administrative records on both PPIs and registry-recorded hospital diagnoses of asthma. The study reported a $57 \%$ increased risk of incident asthma in children exposed to PPI medications. Although the data did not include primary care clinics for the diagnosis of asthma, the study findings are significant and similar to those obtained in our patients with CAD. The biological mechanisms behind the causal relation between PPI use and incident asthma are unclear. PPI medications have been suggested to interfere with the normal digestion of peptides in the stomach, resulting in a T helper 2 dominant response ${ }^{23-25}$. This response is thought to be caused by the preservation of epitopes that are normally degraded by exposure to the acidic environment in the stomach. Alternatively, PPI might directly damage the endothelial function and accelerate endothelial senescence of the lung ${ }^{26}$. This endocrine disruption hypothesis could explain the current finding of an association of PPI use with an increased risk of asthma.

Daily low-dose aspirin is recommended for the prevention of cardiovascular events in patients with CAD, and PPI is recommended to prevent or treat aspirin-associated gastrointestinal injury ${ }^{16-18}$. Previous studies have reported that low-dose aspirin therapy protects against incident asthma ${ }^{27-29}$. However, the current study suggests that PPI treatment increases the risk of asthma development. Therefore, risk of incident asthma should be considered when weighing the benefits and risks of PPI and aspirin treatment in patients with CAD in clinical practice.

Our study is the largest population-based study to examine the association between PPI use and subsequent asthma development. The national database we used contains a representative cohort of 1,000,000 people covered by the Taiwan $\mathrm{NHI}$ program, and the 10-year observation period ensured the power of our statistical analyses. In addition, this is the first population-based study to suggest that PPI use is associated with an increased risk of asthma development in patients with CAD, although some risk factors for PPI are associated with asthma development.

This study has some limitations. First, all patients in this study were collected from claim datasets of Taiwan NHI that had been submitted from primary care clinics. Risk factors for asthma, such as body mass index, smoking status, family history, treatment adherence, environmental tobacco smoke exposure, and diet, were not available from these secondary data. However, considering that we used population-based data, we assumed that no differences exist between PPI users and non-PPI users. Second, all patients in our study sample had received a diagnosis of CAD and peptic ulcer, and received 
PPI treatment. However, dosing, treatment adherence, and severity of CAD with acid-related gastrointestinal tract disorders may have differed across patients who used different PPIs. Thus, the association between PPI therapy and risk for asthma may not reflect the effect of prescribed drugs but rather the severity of the patient's diseases and treatment adherence.

\section{Conclusion}

In conclusion, this large population-based cohort study showed PPI use increased the relative risk of incident asthma in patients with CAD. However, there are no association between PPI use and the risk of incident asthma compared with non-PPI use in patients with hypertension, diabetes mellitus, hyperlipidemia, stroke, urticaria, atopic dermatitis, allergic rhinitis, pneumonia, cancer, or depression. Future clinical studies may help determine if PPI treatment of at-risk patients with CAD could prevent the incident asthma in clinical practice.

\section{Methods}

Study design. This retrospective cohort study used insurance claims data provided by the Taiwanese Bureau of National Health Insurance from January 2004 to December $2013^{30}$. The NHIRD includes the data of information on diagnoses, hospitalizations, examinations, and prescriptions. This study was conducted according to the Declaration of Helsinki and was approved by the Institutional Review Board of Chung Shan Medical University Hospital (IRB No. CS2-20058). Informed consent was also waived by the Institutional Review Board of Chung Shan Medical University Hospital (IRB No. CS2-20058) because of the retrospective nature of this observational study and the anonymous datasets used.

We defined the PPI group in patients with CAD as that newly diagnosed peptic ulcer [International Classification of Diseases, Ninth Revision, Clinical Modification (ICD-9 CM): 531-533] or GERD (ICD-9-CM code 530) between 2004 and 2013. To select the control group, we used 1:1 matching by sex-, age-, and drug index date in subjects who were not PPI prescription. The drug index date of the PPI was defined as the date of the first prescription of PPI in the study period. The endpoint was the development of asthma, which was defined by the time an asthma (ICD-9 CM code: 493) code first appeared in the outpatient claim records.

Study population. Patients at least one of the following inclusion criteria had to be met: (1) two or more outpatient visits within 6 months, (2) all prescriptions of PPI were continuously administered to the patients for more than 3 months within a 10-year follow-up period, or (3) one or more outpatients with a diagnosis of peptic ulcer disease or GERD. Patients who fulfilled any of the following criteria were excluded from the study: (1) had a prior history of asthma before the index date and (2) patients dead within a 10-year follow-up period. Finally, the study group comprised 8,499 participants who were PPI users, and the control group included 8,499 participants who were not PPI users (Figure 1). 
Covariates. Comorbidity was defined as any diagnosis within a year after the drug index date. Asthmarelated comorbidities of patients with hypertension were identified using ICD-9-CM codes 401-405, and hyperlipidemia was identified with ICD-9-CM code 272. Other selected conditions included diabetes mellitus (ICD-9-CM code 250), stroke (ICD-9-CM codes 430-438), urticaria (ICD-9-CM code 708), atopic dermatitis (ICD-9-CM code 691), allergic rhinitis (ICD-9-CM code 477), pneumonia (ICD-9-CM codes 480486), cancer (ICD-9-CM codes 140-208), and depression (ICD-9-CM codes 296, 300, 309, and 311).

Statistical analysis. Data are presented as proportions for the categorical variables. Comparisons between two groups were made using the chi-squared test. The Cox proportional hazard regression model was used to compare the development risk of asthma between PPI and non-PPI users. Adjusted HRs and 95\% Cls were calculated. Their values were adjusted for important risk factors, such as sex, age, urbanization, low income, and comorbidities for asthma development. A Kaplan-Meier plot without covariance correction is presented to analyze the risk of incident asthma according to the presence or absence of asthma. In additional, we conducted a sensitivity analyses to test the robustness of our findings. The propensity score matching (PSMATCH) and inverse probability of treatment weighting (IPTW) was conducted between the two groups by using the SAS software. Finally, we conducted subgroup analyses stratified by sex, age, urbanization, and comorbidities at baseline for the outcomes of incident asthma respectively. All effects were analyzed using an intention-to-treat approach. Statistical significance was considered at $\mathrm{P}<0.05$. All statistical calculations were performed using statistical analysis software, version 9.3 (SAS Institute, Inc., Cary, NC, USA).

\section{Declarations}

\section{Acknowledgements}

The authors gratefully acknowledge expert technical assistance in data management by Dry Lab of the Chung Shan Medical University Hospital.

\section{Author contributions}

C.F.T., T.K.L., L.F.P., and G.P.J. conceived and designed the study, supervised the process of data analysis, and preparation of the manuscript. J.Y.H. contributed to the acquired data and data analysis. G.P.J. wrote the manuscript. All authors commented on, edited, and approved the manuscript.

\section{Competing interests}

The authors have declared that no competing interests exist.

\section{Funding}

This study was supported in parts by grants (TYAFGH-A-110007) from the Taoyuan Armed Forces General Hospital. 


\section{Data availability}

The analysed data in the manuscript is available upon request from the corresponding author.

Authorship note: TKL, CFT, and LFP are co-first authors and contributed equally to this work

\section{References}

1. GBD 2016 Disease and Injury Incidence and Prevalence Collaborators. Prevalence, Global, regional, and national incidence, prevalence, and years lived with disability for 328 diseases and injuries for 195 countries, 1990-2016: a systematic analysis for the Global Burden of Disease Study 2016. Lancet 390, 1211-1259 (2017).

2. Global Health Estimates 2016: Deaths by Cause, Age, Sex, by Country and by Region, 2000-2016. 2018, Geneva, World Health Organization.

3. Prosser, R., Carleton, B. \& Smith, A. The comorbidity burden of the treated asthma patient population in British Columbia. Chronic Dis Can 30: 46-55 (2010).

4. McCallister, J.W. et al. Efficacy of esomeprazole for treatment of poorly controlled asthma. N Eng/ J Med 360, 1487-1499 (2009).

5. Chan, W.W., Chiou, E., Obstein, K.L., Tignor, A.S. \& Whitlock, T.L. The efficacy of proton pump inhibitors for the treatment of asthma in adults: a meta-analysis. Arch Intern Med 171, 620-629 (2011).

6. Kiljander, T.O., Junghard, O., Beckman, O. \& Lind, T. Effect of esomeprazole $40 \mathrm{mg}$ once or twice daily on asthma: a randomized, placebo-controlled study. Am J Respir Crit Care Med 181, 1042-1048 (2010).

7. Kiljander, T.O., Salomaa, E.R., Hietanen, E.K. \& Terho, E.O. Gastroesophageal reflux in asthmatics: A double-blind, placebo-controlled crossover study with omeprazole. Chest 116, 1257-1264 (1999).

8. Kiljander, T.O. et al. Effects of esomeprazole $40 \mathrm{mg}$ twice daily on asthma: a randomized placebocontrolled trial. Am J Respir Crit Care Med 73, 1091-1097 (2006).

9. Peterson, K.A. et al. The role of gastroesophageal reflux in exercise-triggered asthma: a randomized controlled trial. Dig Dis Sci 4, 564-571 (2009).

10. Boeree, M.J., Peters, F.T., Postma, D.S. \& Kleibeuker, J.H. No effects of high-dose omeprazole in patients with severe airway hyperresponsiveness and (a)symptomatic gastro-oesophageal reflux. Eur Respir J 1, 1070-1074 (1998).

11. dos Santos, L.H., Ribeiro, I.O., Sánchez, P.G., Hetzel, J.L., Felicetti, J.C. \& Cardoso, P.F. Evaluation of pantoprazol treatment response of patients with asthma and gastroesophageal reflux: a randomized prospective double-blind placebo-controlled study. J Bras Pneumo/ 3, 119-127 (2007).

12. Gibson, P.G., Henry, R.L. \& Coughlan, J.L. Gastroesophageal reflux treatment for asthma in adults and children. Cochrane Database Syst Rev 2003, CD001496 (2003). 
13. Sopo, S.M., Radzik, D. \& Calvani, M. Does treatment with proton pump inhibitors for gastroesophageal reflux disease (GERD) improve asthma symptoms in children with asthma and GERD? A systematic review. J Investig Allergol Clin Immunol 9: 1-5 (2009).

14. Lai, T. et al. Acid-Suppressive Drug Use During Pregnancy and the Risk of Childhood Asthma: A Metaanalysis. Pediatrics 41, e20170889 (2018).

15. Taqueti, V.R. \& Di Carli, M.F. Coronary microvascular disease pathogenic mechanisms and therapeutic options: JACC state-of-the-art review. J Am Coll Cardiol 72, 2625-2641 (2018).

16. Fortuna, L.A., Pawloski, P.A., Parker, E.D., Trower, N.K. \& Kottke, T.E. Proton pump inhibitor use by aspirin-treated coronary artery disease patients is not associated with increased risk of cardiovascular events. Eur Heart J Cardiovasc Pharmacother 2, 13-19 (2016).

17. Pello Lázaro, A.M. et al. Use of proton-pump inhibitors predicts heart failure and death in patients with coronary artery disease. PLoS One 12, e0169826 (2017).

18. Krill, T. et al. Patients with cirrhosis who have coronary artery disease treated with cardiac stents have high rates of gastrointestinal bleeding, but no increased mortality. Aliment Pharmacol Ther 46, 183-192 (2017).

19. Wang, Y.T., Tsai, M.C., Wang, Y.H. \& Wei, J.C. Association between proton pump inhibitors and asthma: a population-based cohort study. Front Pharmacol 11, 607 (2020).

20. Hak, E., Mulder, B., Schuiling-Veninga, C.C., de Vries, T.W. \& Jick, S.S. Use of acid suppressive drugs in pregnancy and the risk of childhood asthma: bidirectional crossover study using the general practice research database. Drug Saf 36, 1097-1104 (2013).

21. Mulder, B., Schuiling-Veninga, C.C., Bos, H.J., De Vries, T.W., Jick, S.S. \& Hak, E. Prenatal exposure to acid-suppressive drugs and the risk of allergic diseases in the offspring: a cohort study. Clin Exp Allergy 44, 261-269 (2014).

22. Wang, Y.H., Wintzell, V., Ludvigsson, J.F., Svanström, H. \& Pasternak, B. Association between proton pump inhibitor use and risk of asthma in Children. JAMA Pediatr 175, 394-403 (2021).

23. Dehlink, E., Yen, E., Leichtner, A.M., Hait, E.J. \& Fiebiger, E. First evidence of a possible association between gastric acid suppression during pregnancy and childhood asthma: a population-based register study. Clin Exp Allergy 39, 246-253 (2009).

24. Untersmayr, E. \& Jensen-Jarolim, E. The effect of gastric digestion on food allergy. Curr Opin Allergy Clin Immuno/ 6, 214-219 (2006).

25. Bornehag, C.G. \& Nanberg, E. Phthalate exposure and asthma in children. Int J Andro/ 33, 333-345 (2010).

26. Yepuri, G., Sukhovershin, R., Nazari-Shafti, T.Z., Petrascheck, M., Ghebre, Y.T. \& Cooke, J.P. Proton pump inhibitors accelerate endothelial senescence. Circ Res 118, e36-42 (2016).

27. Barr, R.G. et al. Prospective study of acetaminophen use and newly diagnosed asthma among women. Am J Respir Crit Care Med 169, 836-841 (2004). 
28. Kurth, T., Barr, R.G., Gaziano, J.M. \& Buring, J.E. Randomised aspirin assignment and risk of adultonset asthma in the Women's Health Study. Thorax 63, 514-518 (2008).

29. Barr, R.G., Kurth, T., Stampfer, M.J., Buring, J.E., Hennekens, C.H. \& Gaziano, J.M. Aspirin and decreased adult-onset asthma: randomized comparisons from the physicians' health study. $\mathrm{Am} J$ Respir Crit Care Med 175, 120-125 (2007).

30. Chen, H.Y., Huang, J.Y., Siao, W.Z. \& Jong, G.P. The association between SGLT2 inhibitors and newonset arrhythmias: a nationwide population-based longitudinal cohort study. Cardiovasc Diabetol 19, $73(2020)$.

\section{Tables}

Table 1 Baseline characteristics among PPI and non-PPI groups 


\begin{tabular}{|llll|}
\hline & Non- PPI & PPI & p value \\
& $\mathrm{n}=8499$ & $\mathrm{n}=8499$ & \\
\hline Sex & & & 1.0000 \\
\hline Female & $3537(41.62 \%)$ & $3537(41.62 \%)$ & \\
\hline Male & $4962(58.38 \%)$ & $4962(58.38 \%)$ & \\
\hline Age & & & 0.8818 \\
\hline$<20$ & $139(1.64 \%)$ & $133(1.56 \%)$ & \\
\hline 20-45 & $3066(36.08 \%)$ & $3061(36.02 \%)$ & \\
\hline $45-60$ & $2828(33.27 \%)$ & $2858(33.63 \%)$ & \\
\hline 60-75 & $1573(18.51 \%)$ & $1567(18.44 \%)$ & \\
\hline$>=75$ & $893(10.51 \%)$ & $880(10.35 \%)$ & \\
\hline Urbanization & & & 0.0736 \\
\hline Urban & $5015(59.01 \%)$ & $5060(59.54 \%)$ & \\
\hline Sub-urban & $2556(30.07 \%)$ & $2593(30.51 \%)$ & \\
\hline Rural & $928(10.92 \%)$ & $846(9.95 \%)$ & \\
\hline Low income & $55(0.65 \%)$ & $49(0.58 \%)$ & 0.4904 \\
\hline Co-morbidity & & & $<.00001$ \\
\hline Hypertension & $2199(25.87 \%)$ & $2303(27.10 \%)$ & 0.0449 \\
\hline Diabetes mellitus & $915(10.77 \%)$ & $1186(13.95 \%)$ & $<.0001$ \\
\hline Hyperlipidemia & $1271(14.95 \%)$ & $1254(14.75 \%)$ & 0.6901 \\
\hline Stroke & $451(5.31 \%)$ & $712(8.38 \%)$ & $<.0001$ \\
\hline Urticaria & $726(8.54 \%)$ & $536(6.31 \%)$ & $<.0001$ \\
\hline Atopic dermatitis & $148(1.74 \%)$ & $120(1.41 \%)$ & 0.0598 \\
\hline Allergic rhinitis & $1034(12.17 \%)$ & $835(9.82 \%)$ & $<.0001$ \\
\hline Pneumonia & $195(2.29 \%)$ & $380(4.47 \%)$ & $<.0001$ \\
\hline Cancer & & & \\
\hline Depression & & & \\
\hline
\end{tabular}

Table 2 Incidence of asthma in study groups 


\begin{tabular}{|lll|}
\hline & $\begin{array}{l}\text { Non- PPI } \\
\mathrm{n}=8499\end{array}$ & $\mathrm{PPI}$ \\
& $\mathrm{n}=8499$ \\
\hline Follow up person months & 716289 & 719322 \\
\hline Event of asthma & 319 & 387 \\
\hline Incidence rate*(95\% C.I.) & $4.45(3.94-5.01)$ & $5.38(4.87-5.94)$ \\
\hline Crude HR (95\% C.I.) & Reference & $1.21(1.06-1.36)$ \\
\hline aHR (95\% C.I.) & Reference & $1.18(1.05-1.34)$ \\
\hline
\end{tabular}

* Incidence rate, per 10,000 person-months

aHR, adjusted hazard ratio, the co-variates including demographic variables (such as sex, age, urbanization, and low income), and co-morbidities.

Table 3 Sensitivity analysis for the hazard ratio of study events

\begin{tabular}{|lll|}
\hline Model & HR $(95 \% \mathrm{Cl})$ & p value \\
\hline Multiple Cox regression & $1.22(1.08-1.39)$ & 0.0013 \\
\hline IPTW & $1.19(1.06-1.38)$ & 0.0109 \\
\hline Propensity score matching & $1.17(1.04-1.35)$ & 0.0189 \\
\hline
\end{tabular}

Table 4 Subgroup analysis between PPI and non-PPI groups. 


\begin{tabular}{|c|c|c|c|}
\hline \multirow[b]{2}{*}{ Subgroup } & \multicolumn{3}{|c|}{ Patient number/ Incidence rate of asthma } \\
\hline & Non- PPI & PPI & aHR $(95 \% \mathrm{Cl})$ \\
\hline Sex & & & $p$ for interaction $=0.0178$ \\
\hline Female & $3537 / 5.24(4.42-6.18)$ & $3537 / 6.08(5.26-7.02)$ & $1.15(1.01-1.36)$ \\
\hline Male & $4962 / 3.96(3.34-4.68)$ & $4962 / 4.87(4.25-5.59)$ & $1.20(1.06-1.41)$ \\
\hline \multicolumn{4}{|l|}{ Co-morbidity } \\
\hline Hypertension & & & $p$ for interaction $=0.2987$ \\
\hline No & $6300 / 4.52(4.10-4.98)$ & $6196 / 4.13(3.62-4.70)$ & $0.95(0.81-1.13)$ \\
\hline Yes & $2199 / 8.45(7.46-9.58)$ & $2303 / 9.30(7.97-10.84)$ & $1.07(0.88-1.31)$ \\
\hline Diabetes mellitus & & & $p$ for interaction $=0.6381$ \\
\hline No & $7584 / 5.11(4.70-5.55)$ & $7313 / 4.94(4.42-5.52)$ & $1.01(0.88-1.17)$ \\
\hline Yes & $915 / 8.84(7.3-10.71)$ & $1186 / 8.63(6.87-10.84)$ & $0.90(0.67-1.23)$ \\
\hline Hyperlipidemia & & & $\mathrm{p}$ for interaction $=0.4660$ \\
\hline No & $7228 / 5.17(4.75-5.63)$ & $7245 / 4.95(4.42-5.54)$ & $0.98(0.85-1.13)$ \\
\hline Yes & $1271 / 7.25(6.10-8.62)$ & $1254 / 7.89(6.36-9.78)$ & $1.09(0.82-1.44)$ \\
\hline Stroke & & & $p$ for interaction $=0.5184$ \\
\hline No & $8048 / 5.17(4.77-5.6)$ & $7787 / 4.84(4.34-5.39)$ & 0.99(0.86-1.13) \\
\hline Yes & $451 / 12.07(9.45-15.42)$ & $712 / 3.43(10.45-17.26)$ & $1.09(0.76-1.57)$ \\
\hline Urticaria & & & $p$ for interaction $=0.4873$ \\
\hline No & $7773 / 5.27(4.86-5.72)$ & $7963 / 5.18(4.66-5.75)$ & $0.99(0.86-1.13)$ \\
\hline Yes & 726/ 7.62(6.11-9.52) & $536 / 8.37(6.09-11.5)$ & $1.06(0.71-1.57)$ \\
\hline Atopic dermatitis & & & $p$ for interaction $=0.3161$ \\
\hline No & $8351 / 5.46(5.05-5.9)$ & $8379 / 5.33(4.81-5.89)$ & $0.99(0.87-1.12)$ \\
\hline Yes & $148 / 6.29(3.65-10.84)$ & $120 / 9.47(4.93-18.2)$ & $1.61(0.66-3.91)$ \\
\hline Allergic rhinitis & & & $p$ for interaction $=0.9456$ \\
\hline No & $7465 / 5.03(4.62-5.48)$ & $7664 / 5.00(4.48-5.57)$ & $1.00(0.87-1.15)$ \\
\hline Yes & $1034 / 8.67(7.28-10.33)$ & $835 / 8.79(6.88-11.24)$ & $0.99(0.73-1.34)$ \\
\hline Pneumonia & & & $p$ for interaction $=0.7435$ \\
\hline No & $8304 / 5.31(4.91-5.74)$ & $8119 / 5.08(4.57-5.63)$ & $1.00(0.87-1.14)$ \\
\hline
\end{tabular}




\begin{tabular}{|llll|} 
Yes & $195 / 14.49(10.19-20.6)$ & $380 / 16.12(11.4-22.8)$ & $0.95(0.56-1.59)$ \\
\hline Cancer & & & p for interaction= 0.1261 \\
\hline Yes & $8213 / 5.41(5.00-5.85)$ & $7974 / 5.41(4.89-5.99)$ & $1.02(0.89-1.16)$ \\
\hline Depression & $286 / 7.82(5.32-11.48)$ & $525 / 4.70(2.83-7.80)$ & $0.62(0.32-1.22)$ \\
\hline No & & & p for interaction= 0.4473 \\
Yes & $6962 / 5.08(4.65-5.54)$ & $7037 / 4.95(4.42-5.55)$ & $0.97(0.83-1.12)$ \\
\hline
\end{tabular}

\section{Figures}




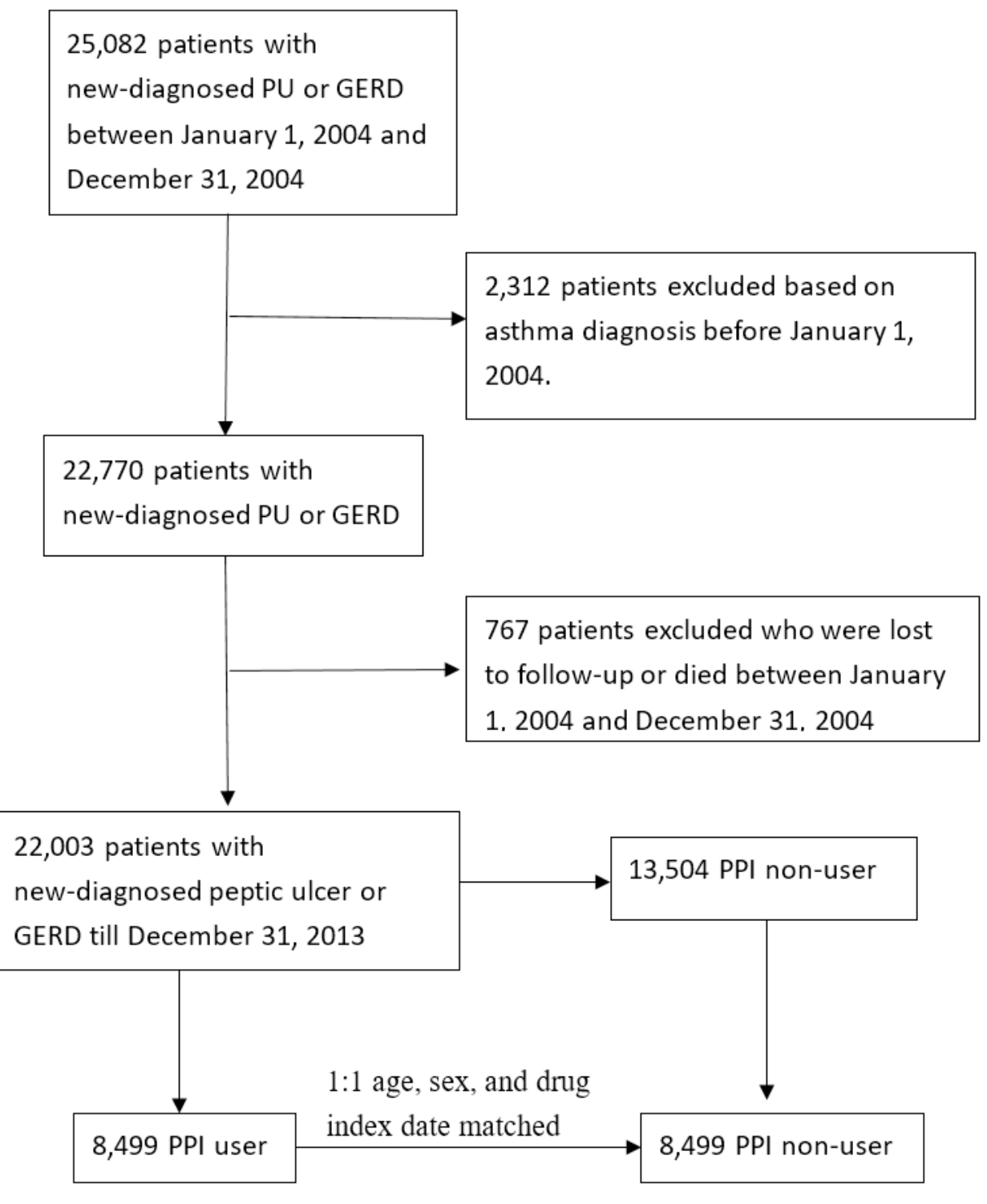

\section{Figure 1}

Patient flow chart. 


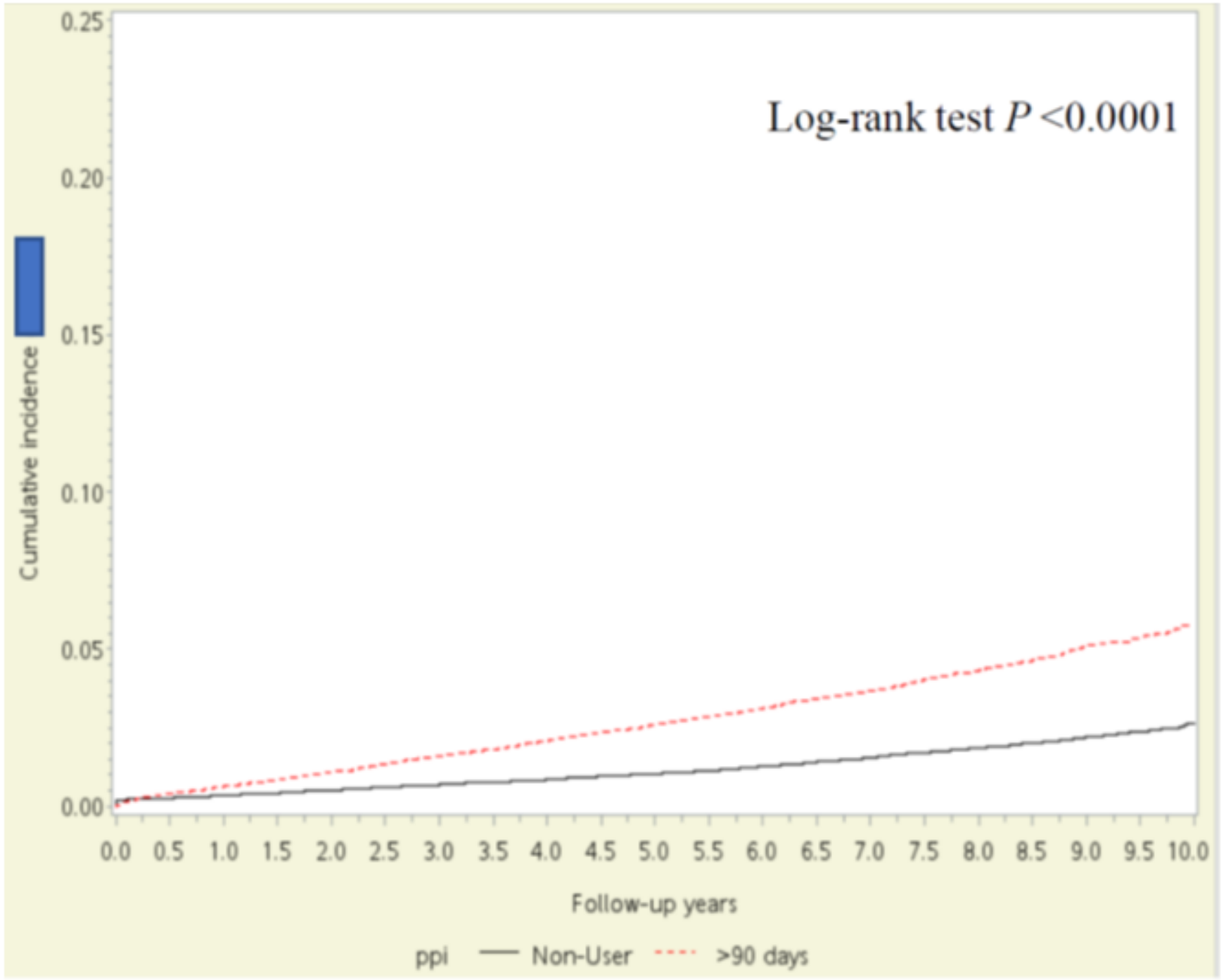

Figure 2

Kaplan-Meier analysis for cumulative incidence of incident asthma between PPI user and PPI non-user group. 